Teorema

Vol. XVI/2, 1997, pp. 97-108

\title{
Philosophy and Mathematics
}

\author{
Andrew Powell \\ Philosophie und Mathematik, by Christian ThIEL, WissenschaftLiche \\ BuchGESELLSCHAFT, DARMSTADT, 1995, 364 pp, 58 DM.
}

This book provides a wide ranging survey of issues in the philosophy of mathematics. It would be an ideal text book to introduce the central themes of the philosophy of mathematics to a reader with a basic background in philosophy and in mathematics. Since, to my knowledge, this book is the only work to provide such a broad introduction to the philosophy of mathematics, it deserves to be successful.

Thiel's book provides good technical and historical coverage of standard material in the philosophy of mathematics: the foundational crisis in mathematics (pp. 315-350); the development of the logicist, formalist, and intuitionist schools (pp. 330-350); the incompleteness results (pp. 221-237); and the structuralism of Bourbaki and Stegmüller (pp. 261-272). It also provides a good introduction to first-order logic in the Hilbert style (pp. 92-102). Rather less standard is the development of logic (pp. 82-85 \& pp. 255-258), arithmetic (pp. 128-138), analysis (pp. 138-155), and geometry (pp. 273-302) in the tradition of Paul Lorenzen, an account of constructive methods (pp. 238-260), and a detailed discussion of the problem of the applicability of mathematics to reality (pp. 30-48). It is to his credit that Thiel does not avoid technicalities. The sketches of the proofs that he gives are for the most part clear and serve to illuminate the issues he discusses.

Both the broadness of the range of the book and the use of technicalities are surely signs of the influence of Thiel's mentor, the sadly late Paul Lorenzen, to whom the book is dedicated. Thiel's book stands in the Lorenzian tradition. For readers unfamiliar with the work of Lorenzen and in order to examine some of the views put forward in this book, I will discuss the Lorenzian tradition later in this review.

As far as general weaknesses are concerned, there are two areas in which the book could be improved.

The first area of weakness is that the bibliographical references are not complete, especially for a survey work of this kind. The references are in fact 
incomplete in two ways: not every text referred to in the book has a reference in the bibliography; and, more generally, certain key works are not referred to at all. An example of the former type of incompleteness is Thiel's reference to Frege's attempt to escape the inconsistency in his Grundgesetze. Although he refers to the literature on this topic (in English) as "Frege's Way Out" (p. 336), he does not cite the paper of the same name by Quine [Mind n.s. 64, pp. 145-159;1955, reprinted in Selected Logic Papers, New York, Random House, 1966; and in Essays on Frege, ed. E.D. Klemke, University of Illinois Press, 1968]. An example of the latter type of incompleteness, again with regard to Frege's way out, is that Thiel does not refer to the work of George Boolos establishing the consistency of the system of Frege's Die Grundlagen der Arithmetik [Foundations of Arithmetic, 1884] relative to a formalization of classical analysis [cf. "The Consistency of Frege's Foundation of Arithmetic", in Being and Saying: Essays in Honor of Richard Cartwright, Cambridge, Mass., M.I.T Press, 1987, pp. 1-20].

The second area of weakness relates to the structure of the book. Each chapter is written as an essay in its own right. Although the chapters are cross referenced and adequately indexed, it is sometimes hard to follow the continuity of thought on any one topic from one chapter to another. By way of an example, consider the topic of David Hilbert's philosophy of mathematics.

The axiomatic method was always central to Hilbert's philosophy of mathematics (and to his mathematical practice), providing a means of clarifying, stabilizing, and systematizing mathematical and scientific thought [cf. "Axiomatisches Denken", Mathematische Annalen, vol. 78, 1918, pp. 405415; Paul Bernays, "Hilberts Untersuchungen über die Grundlagen der Mathematik", Hilberts gesammelte Werke, Berlin, Springer, 1935]. In his Grundlagen der Geometrie [Foundations of Geometry, 1899] Hilbert provided the first axiomatizations of systems of geometry in terms of modern logic. Axiomatic systems also have properties of their own, consistency being the most essential for mathematical thought. In the Grundlagen der Geometrie Hilbert provided consistency proofs for those geometrical systems in terms of analytical models. Since the work of Georg Cantor and Richard Dedekind had shown how to reduce analysis to whole number arithmetic and sets, Hilbert became interested in the problem of the consistency of arithmetic and of set theory. This interest led him (certainly from 1904 onwards) to find properties that a contradiction does not have that the axioms do possess and that the rules of inference preserve. In order to provide a secure foundation for the consistency proofs Hilbert required that the properties can be effectively decided true or false by manipulating finitely many symbols in concrete intuition. Hilbert and his school (most notably Willhelm Ackermann, Paul Bernays, and Gerhard Gentzen) produced consistency proofs by examining the symbolic (or syntactic) properties of proofs. The most sophis- 
ticated example from Hilbert's own school is Gentzen's proof of the consistency of first-order arithmetic. Gentzen showed by transfinite induction (up to a countable ordinal number $\varepsilon_{0}$ ) that every given proof of a contradiction could be effectively simplified to a proof with a lower ordinal number [ $c f$. "New version of the consistency proof for elementary number theory", The Collected Papers of Gerhard Gentzen, Amsterdam, North-Holland, 1969, pp. 252-286]. Since Gentzen formulates mathematical induction as an inference rule, he can take as his basic mathematical axioms free variable formulae which are verifiably true under substitution with symbolic numerals. Hence first-order arithmetic can be proved consistent in a finitistic way (if transfinite induction up to $\varepsilon_{0}$ has a finitistic justification). The view that mathematics is the study of symbolic axiom systems is called formalism; the mathematical study of axiom systems is called meta-mathematics; and the restriction of the methods of meta-mathematics to effectively decidable functions of finitely many symbols is called finitism. Finitistic meta-mathematics is called Hilbertian proof theory (although in the technical sense proof theory nowadays uses non finitistic methods). Hilbert's Programme was to prove the consistency of as much of mathematics as possible by formalizing mathematical systems and providing finitistic consistency proofs. All these terms appear in Thiel's book, formalism (p. 20 \& p. 344), meta-mathematics (p. 72), finitism (p. 242), proof theory (Beweistheorie, p. 242), and Hilbert's Programme (p. 241); and Thiel mentions the origin of Hilbert's Programme (p. 241). However it seems difficult to form an integrated view of Hilbert's philosophy of mathematics from the scattered references in the book. This criticism seems to apply not only to Hilbert but equally to other philosophers of mathematics whose views are discussed in the book.

These general criticisms do not detract greatly from the book as a whole, although they should perhaps be borne in mind in future editions. Before discussing the Lorenzian tradition there is one specific topic on which Thiel's interpretation does not seem quite correct. He says (p. 28, my trans.): "some modern schools of thought (empiricism, logicism, and conventionalism) deny however that there are separate objects [of mathematics] and in this sense that there is an object of mathematics in general". Thiel has previously considered eight modern schools of thought on the nature of mathematical objects: Carl Friedrich Gauss's view of mathematical objects as quantities, Hermann Weyl's view that mathematics is the science of the infinite, Mill's empiricism, the view of Nicholai Hartmann and Günther Jacoby that mathematical objects are fictional things-in-themselves, conventionalism, logicism, formalism, and "structural" formalism. It does not seem correct to say that conventionalism necessarily denies that there are mathematical objects, any more than it is true to say that formalism and "structural" formalism necessarily accept that there are distinctively mathe- 
matical objects. (It is unclear whether mathematics as the science of the infinite admits new mathematical objects or not.)

Conventionalism is the view that there is an element of choice involved when we describe an empirical situation in mathematical terms; that there is a looseness in the fit of mathematical models of experience. A classic example concerns the sum of the angles of a triangle [cf. Hans Reichenbach, The Philosophy of Space and Time, New York, Dover, 1956, p. 33]. If we measure the angles in a triangle, we may say that either the sum of the angles of a triangle is $180^{\circ}$ and that the geometry of space is Euclidean or that there is a force distorting the measuring instruments such that although the sum of the angles of the triangle is still $180^{\circ}$, the geometry is of a non-Euclidean type (in which the sum of angles of a triangle is not $180^{\circ}$ ). While it is true that logicism does not involve any ontological commitments because logic concerns what is true in all possible circumstances (even when there are no individuals), and that empiricism equates mathematical objects with empirical objects, conventionalism does not seem to say what mathematical objects are. The founder of conventionalism, Henri Poincare, held that mathematics is produced from experience by construction and abstraction, but that there are some irreducibly synthetic principles, such as the principle of mathematical induction, and forms of the understanding, such as that of a group of continuous transformations of space, at the base of mathematics [cf. Foundations of Science, New York, Science Press, 1913, p. 64; "On the Foundations of Geometry", The Monist vol. 9, 1898, p. 41]. Reichenbach, on the other hand, held that "the statements of pure geometry hold logically" and that "mathematical geometry ... is analytic" [The Philosophy of Space and Time, p. vi]. When he discusses conventionalism Thiel seems to have Reichenbach's logicism (logical empiricism) in mind rather than Poincaré's own views.

The question of whether formalism has distinctive ontological commitments is less clear cut. The Hilbertian symbolic kind of formalism allows that symbolic axiomatic systems form the ontological commitment of mathematics. But it could be argued that from a formalist standpoint we are ontologically committed to systems of this kind anyway. We all use language, and therefore the grammar or logic of language. If we hold that we can axiomatize segments of our grammar, formalism about mathematics does not introduce any ontological commitments specific to mathematics. A special case is systems of (mathematical) logic. These are axiomatizable, and from a symbolic formalist perspective are the same sort of symbolic game as axiom systems of mathematics. In my view it is for this reason that formalism is inadequate as a philosophy of mathematics; one needs to provide a foundation for the content of the axioms, as explicating the meaning of the logical connectives. 
"Structural" formalism may be regarded as the formalist's way out. "Structural" formalism, structuralism for short, is the view that mathematics concerns the structures which are abstractions from models which satisfy the axioms and rules of inference of the axiom systems of mathematics. Thiel gives an example of the concept of structure by giving a model of the natural sequence (pp. 114-121) and in terms of tallies (|) and a model in terms of circles (in which zero is blank, one is two horizontally adjacent circles, and to form the successor of a number one adds a circle in front of the number and one above it). The tallies and circles which form a "number" can be put into one-to-one correspondence and the successor function for tallies corresponds to the successor function for circles. The natural number structure is then the equivalence relation between such models of the natural numbers (with the stipulation that each model satisfy the principle of mathematical induction for all properties in order to exclude non-standard models of arithmetic). According to structuralism mathematics is ontologically committed to mathematical structures. This is a well established view which has been supported by the approach of twentieth century mathematics. The ontological commitment of structuralism is well defined: equivalence relations (or classes) of models (i.e. sets of objects with relations defined on the objects). But if one asks how one has knowledge of structures then one has to think of a particular spatio-temporal model, unless one is prepared to attribute occult powers to the mind. However, if one decides for reason of ontological parsimony to hold that a spatio-temporal model represents (or is) the structure in certain respects, then one may have no new ontological commitment if one already has an ontology of spatio-temporal objects.

These criticisms are fairly peripheral to the purpose of Thiel's book, so let us proceed to the tradition behind the book, namely to the Lorenzian tradition.

Paul Lorenzen (1915-1994) was a mathematical logician and philosopher of mathematics who built a remarkably original and unified philosophical system. It is a great shame that he is not better known in the Anglo-Saxon countries, although his collected essays are available in English as Constructive Philosophy [Amherst, University of Massachusetts Press, 1987].

Thiel sums up Lorenzen's philosophy as the "interplay of construction and abstraction" [my trans. p. 69, p. 155, p. 313 quoted from Lorenzen's Diffferential und Integral, Frankfurt am Main, Akademische Verlagsgesellschaft, 1965, p. 20]. Lorenzen's philosophical programme was to show how as much of mathematics and the natural sciences as possible could be produced by the process of construction and abstraction. Differential und Integral is in fact the fulfillment of Lorenzen's programme with respect to analysis (the theory of real numbers) using the path developed by Hermann Weyl in Das Kontinuum [Leipzig, Veit, 1918]. Thiel captures the flavour of 
Lorenzen's construction and abstraction process in deriving the real numbers (pp. 128-155), so that a sketch of the derivation seems called for.

The scheme of the derivation is the usual one: real numbers are derived from rational numbers, and rational numbers are derived from natural numbers. The abstraction process itself often makes use of an equivalence relation, i.e. a binary relation $R$ such that $(\forall x) R(x, x),(\forall x, y)(\mathrm{R}(x, y) \rightarrow R(y, x))$, and $(\forall x, y, z)[(R(x, y) \wedge R(y, z)) \rightarrow R(x, z)]$. For an equivalence relation $R$ the abstract of a term $t$ is the property of being equivalent to it, symbolically $(\lambda u) R(t, u)$.

We first construct a sequence of concrete symbols to represent the natural numbers in concrete intuition, say by the rule: $=>|, n=>n|$. Then we define addition and multiplication as (primitive) recursive functions (addition, for example, by $n|+m=(n+m)|$ ). Subtraction and division (on a symbol sequence) can also be defined primitive recursively. Symbols representing negative numbers can then be introduced as an abstraction by means of a primitive recursive definition in terms of the usual arithmetical operations and relations, for instance $-x<0$ if $x>0$. (Thiel actually introduces symbols for negative numbers as an abstraction from a 'recalibration' equivalence relation between ordered pairs of number symbols $(m, n)$ and $(m+k, n+k)$, pp. 136138.)

To form assertions about numbers, we abstract over corresponding symbols. For example, we convert the assertion $A(\|)$ about the symbol $\|$ into the assertion $A^{\prime}(\|)$ about the number which $\|$ represents, as $(\forall x)(x \equiv \| \rightarrow A(x))$, where $\equiv$ is an equivalence relation between two symbols (in the same symbol sequence or not) which holds if the two symbols appear in the same place in a sequence, and where the same symbols for the arithmetical operations are used in each symbol sequence. In this context the universal quantifier should be read substitutionally, namely as "when a symbol equivalent to || is substituted for $x$ then $A(x)$ holds".

Rational numbers are introduced by means of a further abstraction. If we define $(m, n) \equiv(k, l)$ if $1 . m=k . n$ with $n .1 \neq 0$, then $\equiv$ is an equivalence relation. We can then abstract from the representative $(m, n)$ to the rational number $m / n$ (the property of being equivalent to $(m, n)$ ). Real numbers are yet another abstraction. We can define a sequence of rational numbers as a function from the natural numbers to the rational numbers. We cannot construct each sequence of rational numbers: we can construct only those for which $f$ is a recursive function and have to be content with the abstracted concept of sequence of rational numbers. A sequence of rational numbers $f$ is concentrated if $(\forall \varepsilon)(\exists \mathrm{N})(\forall m, n>N)(|f(m) f(n)|<\varepsilon)$, where $\varepsilon$ is a positive rational number, $N, m, n$ are natural numbers, and $|\ldots|$ is the absolute value function $x \Rightarrow x$ if $x>0$ and $x \Rightarrow-x$ if $x<0$. The quantifiers should be read substitutionally, $\exists N$ as "as you can find an $N$ ". A real number can be defined by means of a further 
further equivalence relation: $f=g$ if $(\forall \varepsilon)(\exists N)(\forall n>N)(|f(n)-g(n)|<\varepsilon)$. We can abstract from a concentrated sequence of rational numbers $f$ to the real number which $f$ represents (being the property of being equivalent to $f$ ). Assertions about real numbers are understood as follows. If $A(f)$ is an assertion about a concentrated sequence of rational numbers, then $A^{\prime}(f)$ about the real number which $f$ represents is $(\forall g)(g \equiv f \rightarrow A(g))$.

Now that we have sketched the derivation of real numbers from natural numbers, there are some points about the method which are characteristic of Lorenzen and his school.

The first point is that the basis of the derivation is everyday experience, concrete symbols (albeit regulated by a conceptual requirement that we recognize symbols as of the same kind, for instance one $\mid$ and another $\mid$ ) and commonplace operations on the symbols. The use of everyday experience as the ultimate reference for mathematics and natural science closes the epistemological gap present in many philosophies of mathematics. For Lorenzen this requirement gives rise to a proto subject, which is the subject before the process of construction and abstraction begins.

The second point is that Lorenzen's approach is always based on constructions. Lorenzen seems to have regarded part of mathematics or natural science as unfounded if it could not be founded by means of constructions. In Differential und Integral Lorenzen followed Weyl in rejecting real numbers formed with reference to the totality of all real numbers, permitting instead only that arithmetical properties of real numbers could be used. A good deal of real analysis can then be developed [cf. Gaishi Takeuti, Two Applications of Logic to Mathematics, Tokyo, I. Shoku; and Solomon Feferman, "Weyl vindicated: 'Das Kontinuum' 70 years later", Temi e prospetti della logica e della filosofia dell scienzia contemporanea, vol. 56, Bologna, CLUEB, 1990, pp. 59-93 for alternative developments]. The classical assertion of completeness that every set of real numbers with an upper bound has a least upper bound then fails; but we can instead assert that every sequence of real numbers with an upper bound has a least upper bound.

The third point is that Lorenzen's construction and abstraction process has a strong nominalist flavour. From the beginning with concrete particulars to a process designed to minimize the ontological burden of the final result. Although the immediate source is probably the influence of Immanuel Kant, of which more below, the method employed by Lorenzen is in keeping with the spirit of William of Ockham and the via moderna.

The fourth and final point is that the strongest influence on Lorenzen's abstraction and construction process seems to be Kant. As Thiel notes (p. 27) the Kantian method in mathematics was to construct a mathematical concept in intuition, this statement applying equally to geometry, arithmetic and algebra [cf. Critique of Pure Reason, A717/B745 and A734/B762]. In his Logic 
[1800, trans. R.S. Hartmann \& W. Scherz, Indianopolis, Bobbs-Merrill, 1974, s6, pp. 100-101] Kant gave the principles of formation of empirical concepts as comparison, reflection and abstraction: we compare the features of things, reflect on what they have in common, and finally abstract away the features not in common. This process is similar to the abstraction process envisaged by Lorenzen: the only difference is that when you have an equivalence relation you have already removed what is different between them. It seems that the similarity between Kant and Lorenzen goes even further. The construction process begins with everyday experience. But, as noted above, to be more exact it begins with spatio-temporal objects subjected to certain common concepts. Kant's view of mathematical concepts seems to have been that for spatio-temporal objects to be treated as mathematical they need to be constructed in intuition insofar as they satisfy certain concepts of the understanding. For instance, a number is "a representation which comprises the successive addition of homogeneous units" [Critique of Pure Reason, A142143/B182]. It seems reasonable to suppose that the homogenous units have a concept as a ground of their unity. On this interpretation to judge that "there are five apples" you firstly judge each apple under the concept "apple", and then apply the counting rule $(=>|, n=>n|)$ to synthesize the five units as a single totality. Lorenzen's approach seems very similar to this interpretation of Kant's views on mathematical concepts.

To test the interpretation of Lorenzen's philosophy given above, let us consider the foundations of geometry and logic.

In the Lorenzian tradition geometry is the theory of spatial form. We begin from the everyday rules that we have learnt about spatial objects: how they match one another, and how they fit together. This is protogeometry. Lorenzen believed that Euclidean geometry is privileged because it can be given a foundation in terms of the abstraction and construction process. The account given here is based on Lorenzen's article "The Foundational Problem of Geometry" [in Constructive Philosophy, pp. 257-273] with some assistance on dimensionality from Bernard Riemann's "On the Hypotheses which Lie at the Foundation of Geometry" [A Source Book in Mathematics, New York, McGraw-Hill, 1929, pp. 411-425].

We firstly need some definitions. An extension is a connected spatial object which can be taken to consist of as many parts as one pleases. One extension $X$ is said to be homogeneous with respect to an extension $Y$ if every part of $X$ has the same properties with respect to $Y$. A spatial object is said to be homogenous if it is homogeneous with respect to itself. Let us characterize some geometrical terms. A point is an extension which we stipulate can be mapped onto itself only by the identity map. A line is an extension which can be mapped onto itself by two mutually inverse families of maps. A plane is an extension which can mapped onto itself with two pairs of mutually inverse 
families of maps (with the independence property, that the only map in both pairs is the identity map). A space is an extension which can be mapped onto itself by three independent pairs of mutually inverse families of maps. A plane is Euclidean if it is homogeneous with respect to the space in which it is embedded. (We identify coincident parts on the top and the bottom of the plane.) A highly symmetric body like the sphere fails to be a Euclidean plane because when embedded in space one can distinguish a top and a bottom. Because we cannot distinguish edges of the Euclidean plane a Euclidean plane must be unbounded in every direction. A line is Euclidean if it is formed by the intersection of two Euclidean planes. Two Euclidean planes (lines) are parallel if every point on one plane (line) is homogeneous with respect to every point on the other plane (line). Parallel Euclidean planes (lines) cannot intersect because the line (point) of intersection would be distinguished. A Euclidean line is orthogonal to a Euclidean plane if every Euclidean line in the plane that passes through the point of intersection of the plane and the line is homogeneous with respect to the point. The Euclidean axiom of parallels is true because given any Euclidean line and a point not on the line we can construct parallel Euclidean planes containing the point and the line. Moreover, the Euclidean planes are parallel in the normal sense: any Euclidean line orthogonal to one Euclidean plane is orthogonal to the other. We can see this by imagining that there were two Euclidean lines, $L 1$ and $L 2$, through a point on one Euclidean plane, $E, L 1$ orthogonal to that Euclidean plane and the other $L 2$ orthogonal to the other Euclidean plane, $F$. Then the Euclidean plane containing $L 1$ and $L 2$ will intersect the $F$ in a particular line. But this line is distinguished with respect to the point of intersection of $L 2$ and $F$. This contradicts the definition of orthogonality of $L 2$ and $F$. Hence $L 1$ is identical to $L 2$. We can complete the construction of Euclidean geometry by showing how Euclidean congruence can be defined using parallelism and orthogonality, because we can define a rigid motion as one that preserves Euclidean congruence. We omit the details here, but refer the interested reader to Lorenzen's original article.

One significant feature of this account is the abstraction involved in forming the notion of Euclidean plane. After all no actual spatial object is exactly homogeneous with respect to its embedding space. The important point is that homogeneity is a logical notion (based on the identity of indiscernibles). When applied to Euclidean geometry homogeneity (of $X$ with respect to $Y$ ) is an equivalence relation, thus giving rise to a foundational account of Euclidean geometry which mirrors that of the theory of real numbers. The constructive element arises because geometrical objects must be constructed in the original Euclidean (and Kantian) sense.

Before discussing Lorenzen's foundation of logic, it is worthwhile to remark that Lorenzen's philosophical approach has a strong sense of history: 
his article on geometry can be thought of as a defence of Euclid's original geometrical spirit.

Just as geometry begins with protogeometry, logic begins with protologic. Protologic might be defined as logic that arises out of considerations of practice or usage prior to logic. With respect to mathematics such considerations are syntactic operations on proofs, since truth values for quantified formulae with infinite domains involve us in adopting infinities as actual or completed totalities. Thiel gives an example (pp. 82-83). The introduction rules for the logical "or" connective are: $a \rightarrow a \vee b$ and $b \rightarrow a \vee b$. If we could assert $a \rightarrow c$ and $b \rightarrow c$, then if we could assert $a \vee b$ then we could assert $c$. This is so because $a \vee b$ must have been introduced by either $a \rightarrow a \vee b$ or $b \rightarrow a \vee b$; so we can use either the proof of $a \rightarrow c$ or the proof of $b \rightarrow c$ to prove $c$. Thus we have justified $[(a \rightarrow c) \wedge(b \rightarrow c)] \rightarrow(a \vee b \rightarrow c)$. This last fomula can be seen as a rule for eliminating the logical "or" connective $\vee$, if we write it as $a \vee b, a \rightarrow c, b \rightarrow c \Rightarrow c$, where $\Rightarrow$ stands for "is inferred from". Using ideas of natural deduction developed originally by Gerhard Gentzen and enhanced by Dag Prawitz [cf. "Ideas and Results of Proof Theory", in ed. J. Fenstad, Proceedings of the Second Scandinavian Logic Symposium, Amsterdam, NorthHolland, 1973, pp. 235-306] it is possible to formulate logical systems by providing introduction rules and elimination rules for each logical connective. One can provide at least a partial foundation for logic if we can justify an elimination rule as a consequence of its introduction rule, and say that a logical connective is defined by its introduction rule [cf. "Ideas and Results of Proof Theory" p. 247]. The logic that we can found in this way is called minimal logic, which is a subsystem of both intutionistic and classical logic (cf. "Ideas and Results of Proof Theory" p. 240). If we wanted to justify intuitionistic logic we would need to justify the ex falso quodlibet inference of any atomic proposition from the assertion of a contradiction; and for classical logic we would need a justification of double negation elimination.

With Kuno Lorenz Lorenzen also developed dialogic [cf. Dialogische Logik, Wissenschaftliche Buchgesellschaft, Darmstadt, 1978], a formulation of logical systems as a dialogue between a proponent and an opponent, each of whom tries to win the dialogue by forcing the other into a contradiction. This approach to logic once again seems to have its origin in the history of philosophy, in particular in the Socratic method.

It is very difficult to evaluate a whole tradition, but I find Lorenzen's foundational work in the mathematics far more satisfactory than his foundations of logic. Usage-theoretic views of the foundation of logic are deeply unsatisfactory philosophically: for, in what do you ground your usage? In the case of mathematics "usage" becomes "proof". At some stage one must give some meaning-theoretic justification of some logical rules or axioms; and it is circular to justify these rules or axioms on some prior notion of proof. Dia- 
logic does not seem any better: for, what is the ground of the rules employed in a dialogical dispute? My own view is that each domain of discourse may have its own grammar or logic, and that logic needs to be founded in terms of a particular standpoint that you consider legitimate, in mathematics the finitistic standpoint, the potential infinite standpoint, or the actual infinite standpoint, for example.

Thiel's book fits well into the Lorenzian tradition. He follows Lorenzen in his discussions of arithmetic and analysis, and in geometry (pp. 273-302) describes a variant of Lorenzen's approach due to Rüdiger Inhetveen [Konstruktive Geometrie, Mannheim, B.I.-Wissenschaftsverlag, 1983]. As I understand it, the Euclidean plane is specified by the property that if any part of the plane is brought into contact with another part by a fold the parts on the plane will fit together exactly. This property is certainly an homogeneity condition on the Euclidean plane in terms of its embedding space. The concept of orthogonality is founded by having two wedges of opposite orientation which fit together exactly; and parallelism arises when two "pieces of cake" bodies are brought into contact, the non-touching straight sides being parallel.

As I mentioned at the start of this review, Thiel discusses the problem of the applicability of mathematics to reality. As Thiel notes (p. 40) the applicability problem is a neglected area of the philosophy of mathematics. It is also an area of central importance to the subject, because mathematics is altogether mysterious if it is a domain its own right which just happens to be essential in formulating most physical laws. Lorenzen's foundations of analysis and geometry are formulated to solve the applicability problem: mathematics is applicable to reality because and insofar as it is constructed from reality. It seems that the Lorenzian tradition led Thiel into a discussion of the applicability problem.

Thiel analyses the difference between empiricism and Kantianism by saying that the empiricist view is that mathematics is applicable to reality because it originally depends on reality, while Kant's view was that mathematics is applicable to reality because both reality and mathematics depend on the same laws (p. 37). Thiel also gives a very interesting account of the work of Victor Kraft, who was the member of the Vienna Circle who gave the deepest analysis of the applicability problem. (This was a particular problem for the logical empiricists because they held that since "pure" mathematics was not empirical it must be logical or analytic.)

There are some elements in Thiel's work that do not seem inspired by Lorenzen. Thiel's discussion of the foundational crisis in mathematics in the early part of this century (pp. 330-350) has a disengaged, methodological flavour that I have not found in Lorenzen's. work. Thiel stands back from the subject and looks at the subsequent developments with the eye of an historian and methodologist of the philosophy of mathematics. 
Thiel also seems more in sympathy with the structuralist position than does Lorenzen. Thiel says (p. 314, my trans.):

That we speak inter alia of "sets of" and refer with points to meaningful sorts of mathematical objects already suggests in fact that we do indeed perform the same operations in different areas of mathematics, but that there are no 'sets' as autonomous objects which form a proper category or have the objects 'in themselves' for which the type of the expression is referred to at the location of the points.

Lorenzen was not a structuralist nor an advocate of general set theory. In fact in order to admit theories which admit the law of the excluded middle to a theory with quantification over an infinite domain, in his inaugural lecture at the University of Kiel in 1957 "Wie ist Philosophie der Mathematik möglich?" ["How is Philosophy of Mathematics possible?", published in Philosophia Naturalis IV, 1957, pp. 192-208] Lorenzen required a consistency proof from the finite standpoint [ $c f$. Hilbert, "Beweis des Tertiums non datur", Nachrichten von der Gesellschaft der Wissenschaft zu Göttingen, 1931, pp. 120-125]. Thiel does not seem to require consistency proofs for theories of the infinite structures of classical mathematics.

Thiel's discussion of logic has elements of Lorenzen's work in protologic and dialogic. I was unaware of the result (p. 258) that classical logical propositional truths are truths which can established by a proponent in a dialogue using knowledge of the truth or falsehood of the propositions that make up the truth, while intuitionistic logic can be established by the proponent independently of the truth or falsehood of those propositions. This is a very interesting result. But Thiel also presents a nice formulation of systems of propositional logic in the Hilbertian style (pp. 92-102). The derivation of double negation elimination on pp. 100-102 can possibly be shortened. Step (22) $\neg \neg p \rightarrow(\neg \neg p \rightarrow p)$ can be derived by noting that $\neg \neg p \rightarrow(\neg \neg \neg \neg p \rightarrow \neg \neg p)$ is an axiom and $(\neg \neg \neg \neg p \rightarrow \neg \neg p) \rightarrow(\neg \neg p \rightarrow p)$ follows by applying the axiom $(\neg q \rightarrow \neg p) \rightarrow(p \rightarrow q)$ twice with suitable substitutions.

Bearing in mind these differences, Thiel's work does make a worthy contribution to the Lorenzian tradition, and is well worth reading on those grounds and in its own right.

Department of Philosophy

King's College London

The Strand

London WC2R 2LS UK 